\title{
The long-term optical spectral variability of BL Lacertae ${ }^{\star}$
}

\author{
I. E. Papadakis ${ }^{1,2}$, M. Villata ${ }^{3}$, and C. M. Raiteri ${ }^{3}$ \\ 1 Physics Department, University of Crete, PO Box 2208, 71003 Heraklion, Crete, Greece \\ e-mail: jhep@physics.uoc.gr \\ 2 IESL-Foundation for Research and Technology, 71110 Heraklion, Greece \\ 3 INAF-Osservatorio Astronomico di Torino, via Osservatorio 20, 10025 Pino Torinese (TO), Italy
}

Received 21 March 2007 / Accepted 4 May 2007

\begin{abstract}
Context. We present the results from a study of the long-term optical spectral variations of BL Lacertae, using the long and wellsampled $B$ and $R$-band light curves of the Whole Earth Blazar Telescope (WEBT) collaboration, binned in time intervals of 1 day. Aims. We study the relation between the long-term spectral variations and the respective flux variations of the source. Methods. Using cross-correlation techniques to investigate whether there are any delays between the flux variations in different energy bands and between the flux and spectral variations.

Results. The relation between spectral slope and flux (the spectrum gets bluer as the source flux increases) is well described by a power-law model, although there is significant scatter around the best-fitting model line. To some extent, this is due to the spectral evolution of the source (along well-defined loop-like structures) during low-amplitude events, which are superimposed on the major optical flares, and evolve on time scales of a few days. The $B$ and $R$-band variations are well correlated, with no significant, measurable delays larger than a few days. On the other hand, we find that the spectral variations lead those in the flux light curves by $\sim 4$ days. During at least the largest amplitude flares, the $B$-band variations appear to evolve faster than those in the $R$ band.

Conclusions. We confirm the "bluer-when-brighter" mild chromatism of the long-term variations, and we show that it can be explained if the flux increases/decreases faster in the $B$ than in the $R$ band. We also report the discovery of the lag between spectral and flux changes. These two features can be explained in terms of Doppler factor variations due to changes in the viewing angle of a curved and inhomogeneous emitting jet.
\end{abstract}

Key words. galaxies: active - galaxies: quasars: general - galaxies: jets - galaxies: BL Lacertae objects: general galaxies: BL Lacertae objects: individual: BL Lacertae

\section{Introduction}

BL Lacertae is the prototype of a class of active galactic nuclei (AGNs) known as "BL Lac objects", or simply "BL Lacs". The members of this class show non-thermal continuum energy spectra, fast and large-amplitude variations from radio up to $\gamma$-rays, a high degree of linear polarization, and radio jets with individual components often exhibiting apparent superluminal motion.

The "Whole Earth Blazar Telescope" (WEBT; http://www.to.astro.it/blazars/webt/) is a large international collaboration among optical and radio astronomers, established in 1997. It organizes monitoring campaigns on selected blazars in order to obtain continuous, high-temporaldensity light curves in the optical and radio bands (usually in conjunction with observations at other wavelengths like $\mathrm{X}$ and $\gamma$-rays; see e.g. Böttcher et al. 2005; Raiteri et al. 2005, 2006; Ostorero et al. 2006; Villata et al. 2006, 2007).

BL Lacertae has been the target of four WEBT monitoring campaigns in the past. The first two were rather short. They were organized in 1999, simultaneously with $A S C A$ and BeppoSAX observations (Ravasio et al. 2002). The remaining two were long-term campaigns carried out in the periods May 2000-January 2001 (Villata et al. 2002; Böttcher et al. 2003) and May 2001-February 2002 (Villata et al. 2004b,a).

* Based on data taken and assembled by the WEBT collaboration and stored in the WEBT archive at the Osservatorio Astronomico di Torino INAF.
Both campaigns resulted in long, well-sampled, and highprecision light curves.

Using data from the first long-term WEBT campaign, Villata et al. (2002) found that the $B$ and $R$-band light curves were well correlated with no measurable time delay. They also found that the flux variations are associated with significant spectral variations. They interpreted them in the context of a two variability mechanism model: the first mechanism is essentially achromatic and it is responsible for the long-term variations (i.e. variations which operate on time scales of a few days), while the second one causes fast (i.e. shorter than a day) flares, superposed on the long-term variations, and introduces spectral changes in the sense that the spectrum becomes harder (bluer) as the source brightens.

Similar results were obtained by Villata et al. (2004b), who studied not only the light curves from the 2001-2002 WEBT campaign, but also composed light curves from 1994 to 2002 , in all optical bands, with data taken from the literature as well as by members of the WEBT. The authors distinguished a "mildly-chromatic" (in the sense that the $B-R$ versus $R$ plot has a slope $\sim 0.1$ ), long-term variability component, which operates on time scales longer than a few days, from a "strongly-chromatic" (in which the variations trace a 0.4 slope in the $B-R$ versus $R$ plot), short-term variability mechanism, which operates on intra-day time scales.

Villata et al. (2004b) based their results mainly on the study of the best-sampled parts of the light curves, which cover the 
period between 1997 and 2002. In this work, we focus our attention on the $B$ and $R$-band light curves of BL Lacertae in the same period. Our main aim is to better understand the long-term chromatic behaviour of the source, and to investigate possible reasons for the observed spectral variations.

In Sect. 2 we present the light curves we use in this work and we quantify the "spectral slope versus flux" relation on time scales longer than a day. Section 3 deals with the crosscorrelation analysis between the $B$ and $R$-band light curves, and between the spectral slope and flux variations. In Sect. 4 we present the results from the study of two long, well sampled, large-amplitude flares in the light curves, while our final conclusions are discussed in Sect. 5.

\section{The optical light curves}

Villata et al. (2004b) presented UBVRI light curves of BL Lacertae from 1994 up to 2002, using data from the four WEBT campaigns and the literature. The host galaxy constant flux contribution was subtracted from the observed light curves in all bands as explained in Sect. 5 of Villata et al. (2002). We chose to use the Villata et al. (2004b) $B$ and $R$-band light curves from the period between 1997 and February 2002, as we believe that these are the "best" light curves (in terms of length, number of data points and sampling frequency of observations) among the currently available optical datasets of this object. The data that we use in this work are now publicly available in the WEBT archive (http://www . to. astro.it/blazars/webt/) and can be requested by sending a message to the WEBT president.

In order to minimize any systematic effects that could influence our results we examined carefully the Villata et al. (2004b) data and selected our final $B$ and $R$-band datasets as follows: a) we kept data with errors not greater than 0.04 and 0.03 mag in the $B$ and $R$-band light curves; b) we used only those data points which have at least one counterpart in the other band within $20 \mathrm{~min}$, either from the same telescope or from the same literature paper; c) we also removed three "unreliable" $B$ data points.

The two upper panels in Fig. 1 show the $B$ and $R$-band light curves (in flux density units) that we use in this work. Since we are interested in studying the spectral variations on time scales longer than a day, the light curves are binned in 1-day long intervals, starting from JD $=2450644$. If more than one point is present in the interval, we take their average flux density and time of observation. We consider the standard deviation, i.e. $1 \sigma$, the average spread of the points around their mean, as the "error" on the average flux density in the respective bin. In the case where there is just one point in a bin we accept its error as it is. Typically, the error of the points, estimated in this way, in the $B$ and $R$ band 1 day binned light curves is of the order of $\sim 0.1-1 \mathrm{mJy}$. Therefore, although we do plot the error bars of the points in Fig. 1, most of them are smaller than the size of the symbols we use.

The source is brighter in the $R$ band. The maximum flux density recorded in that band is almost twice the respective maximum flux density in the $B$-band light curve. Apart from this difference, the two light curves look very similar. The same variations appear, simultaneously, in both of them. However, the amplitude of the observed variations is larger in the $B$-band light curve. Indeed, the "fractional variability amplitude", $f_{\text {rms }}$ (defined as the ratio of the standard deviation over the light curve mean, see Papadakis et al. 2003), of the $B$ and $R$ light curves is $65.3 \pm 0.2 \%$ and $57.1 \pm 0.2 \%$, respectively. The errors
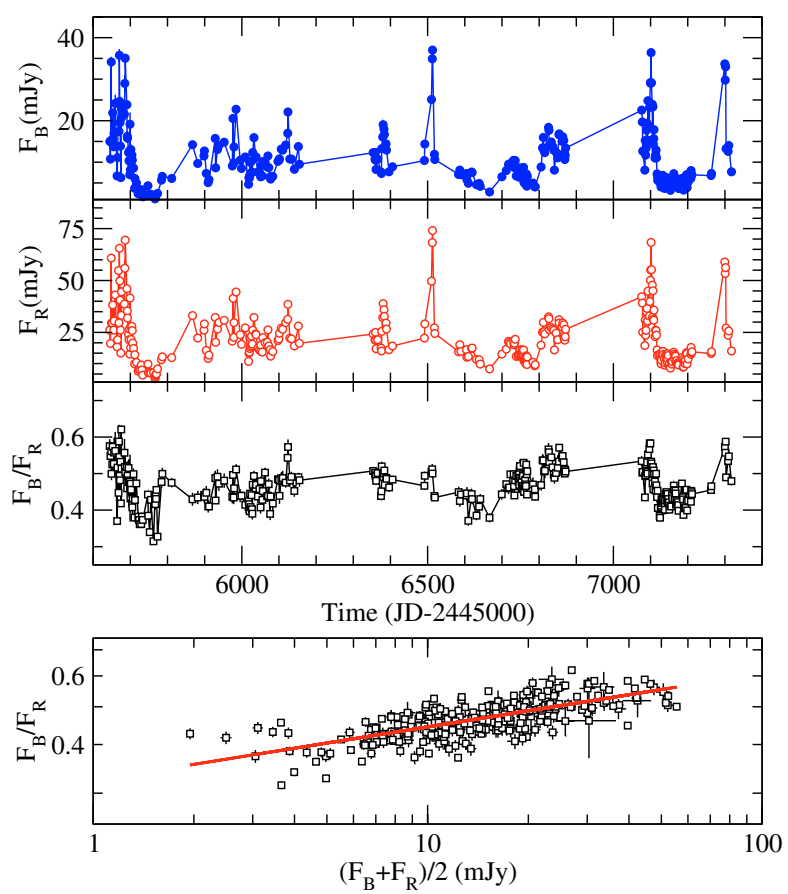

Fig. 1. First and second panel from top: the 1997-2002 B and $R$ light curves of BL Lacertae binned in intervals of size equal to 1 day. Third panel from top: the $F_{B} / F_{R}$ flux density ratio, plotted as a function of time. Bottom panel: $F_{B} / F_{R}$ plotted as a function of $\left(F_{B}+F_{R}\right) / 2$. The solid line shows the best fitting power-law model to the data.

of $f_{\mathrm{rms}}$ account only for the measurement errors in the light curve points, and have been estimated according to the prescription of Vaughan et al. (2003). Given the very good correlation between the $B$ and $R$ band light curves, one could assume that the $\sim 7 \%$ difference in $f_{\mathrm{rms}, B}$ and $f_{\mathrm{rms}, R} B$ maybe due to differences in the experimental noise level of the two light curves. For example, Poisson noise will introduce some scatter in the $f_{\text {rms }}$ values we measure. However, the errors we provide (following Vaughan et al. 2003) suggest that this is not the case. The difference between the $B$ and $R$ band variability amplitudes is most probably an intrinsic property of the variability mechanism that opearates in the source.

\subsection{Spectral variations}

The third panel from the top in Fig. 1 shows a plot of the $F_{B} / F_{R}$ flux density ratio as a function of time. This ratio can be considered as representative of the slope of the optical spectrum of the source. Errors on this ratio have been estimated using the usual "propagation of errors" recipe (see e.g. Bevington 1969).

Since the $B$ and $R$-band light curves do not have the same fractional variability amplitude, we expect the spectral slope (and hence the ratio $F_{B} / F_{R}$ ) to change with time. Indeed, Fig. 1 shows clearly that this ratio is highly variable. The fractional amplitude, $f_{\mathrm{rms}, R}$, of the $F_{B} / F_{R}$ variations is $10.3 \pm 0.2 \%$ (where the error accounts only for the measurement error of the $F_{B} / F_{R}$ points, as explained above). We conclude that, on time scales longer than 1 day, the optical flux density variations of BL Lacertae are associated with moderate, but significant, spectral slope variations as well.

In the bottom panel of Fig. 1 we plot the $F_{B} / F_{R}$ ratio as a function of $\left(F_{B}+F_{R}\right) / 2$, i.e. the mean of the $B$ and $R$-band flux densities, which can be considered as representative of the flux density in an intermediate band. We have chosen to use 
this quantity in the study of the "spectral slope $\left(F_{B} / F_{R}\right)$ versus brightness" relation, as the average of the source signal in the two bands should minimize the possibility of introducing artificial correlations in this relation. Such correlations could be introduced if we were using either the $B$ or the $R$-band flux densities, individually, because of the interdependency of the $F_{B} / F_{R}$ values and these measurements.

The "spectral slope vs. brightness" plot shows clearly that the flux and spectral variations are well correlated: as the source flux increases (decreases), the spectrum becomes harder (softer). The solid line in the bottom panel of Fig. 1 shows the best fitting power-law (PL) model to the data: $F_{B} / F_{R} \propto\left[\left(F_{B}+\right.\right.$ $\left.\left.F_{R}\right) / 2\right]^{0.14 \pm 0.01}$. Although it does not provide a statistically acceptable fit (we get a $\chi^{2}$ value of 6313.5 for 308 degrees of freedom when we consider the measurement uncertainty of each point in the plot), it nevertheless describes rather well the overall trend of the $F_{B} / F_{R}$ increase with increasing flux.

The statistically poor quality of the fit is due to the fact that there exists a significant scatter in the $F_{B} / F_{R}$ values for a given flux density. However, it is of small amplitude (at each flux level, the maximum deviation around $\left(F_{B} / F_{R}\right)_{\text {model }}$ is less than $\sim \pm 15 \%$ of $\left.\left(F_{B} / F_{R}\right)_{\text {model }}\right)$ and does not show any systematic deviation from the best fitting PL model. This scatter implies some residual, weak spectral variability which is not correlated with the flux density level of the source.

Interestingly, the $F_{B} / F_{R} \propto\left[\left(F_{B}+F_{R}\right) / 2\right]^{0.14}$ relation would correspond to a line of slope $\sim 0.14$ in a " $B-R$ vs. magnitude of $\left(F_{B}+F_{R}\right) / 2$ " plot. This is close to the "softer" slope of 0.1 in the " $B-R$ vs. $R$ " plot of Villata et al. (2004b). Hence the flux-density variations that we observe in the 1-day binned light curves of Fig. 1 correspond to the "mildly-chromatic", long-term variations (Villata et al. 2004; see also Hu et al. 2006).

\section{Cross-correlation analysis}

\subsection{Correlation between the $B$ and $R$-band light curves}

A possible explanation for the observed spectral variations is the existence of a delay between the $B$ and $R$-band light curves: if the flux increases or decreases with the same rate in both light curves, but the $B$-band variations lead those in the $R$ band, then the spectrum is "bluer"/"redder" when the flux is rising/decaying.

In order to investigate this possibility we used the discrete correlation function (DCF) method of Edelson \& Krolik (1988). Our results, with a DCF bin size of 4 days, are plotted in the top panel of Fig. 2 (open circles). We get similar results when we use a wide range of DCF bin sizes, from 1 to 10 days. A positive lag of the DCF peak in this plot would imply that the $B$-band variations lead those in the $R$ band. In the same panel we also plot the $B$-band auto-correlation function (ACF; solid line). The ACF of the $F_{R}$ and $\left(F_{B}+F_{R}\right) / 2$ light curves are practically identical to that of the $F_{B}$ light curve.

The $F_{B}$ vs. $F_{R}$ DCF is similar to the $B$-band ACF and shows a strong $\left(\mathrm{DCF}_{\max } \approx 1\right)$ and narrow peak at zero time lag. Strong and narrow DCF maxima at zero lags can be introduced when systematic errors affect, in the same way, the data points in both light curves. However, in our case, the points in the light curves shown in Fig. 1 correspond to observations from many different telescopes, so that the presence of a global systematic effect is rather unlikely.

If we omit the DCF point at zero lag, then the highest DCF value is $\sim 0.7$. If we now estimate the centroid lag, $\tau_{\text {cent }}$, using all DCF points with values in excess of $0.7 \mathrm{DCF}_{\max }$

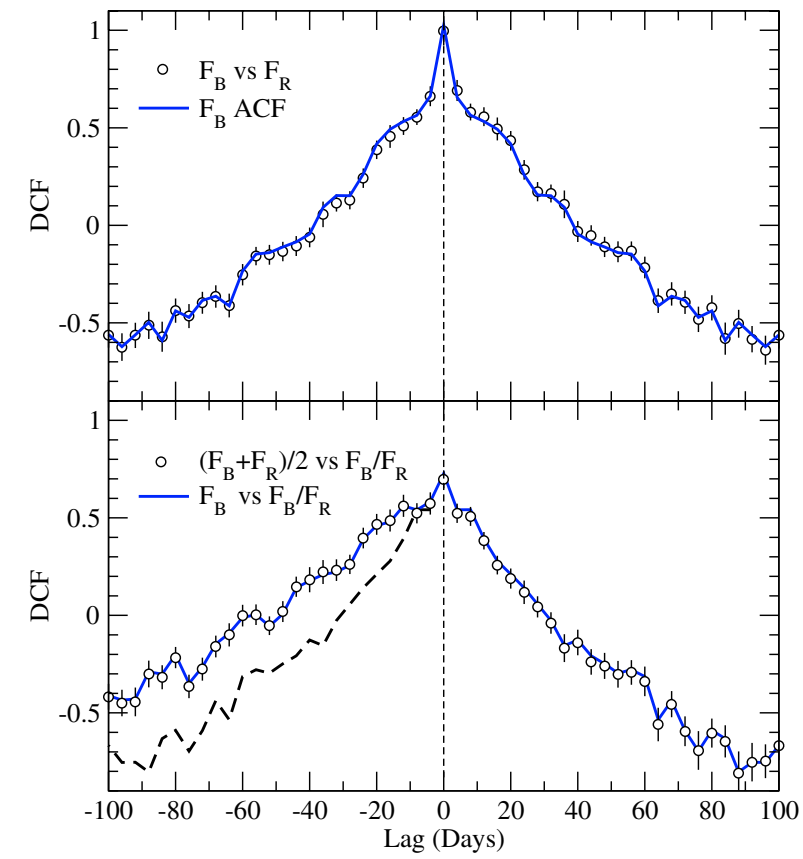

Fig. 2. Top panel: the $B$-band ACF (solid line) and the $F_{B}$ vs. $F_{R}$ DCF (open circles). Bottom panel: the $\left(F_{B}+F_{R}\right) / 2$ and $F_{B}$ vs. $F_{B} / F_{R}$ DCFs (open circles and solid line, respectively). The positive-lag $F_{B}$ vs. $F_{B} / F_{R}$ DCF part is reflected about the zero-lag axis (dashed line) for comparison (see text for details).

(assuming $\mathrm{DCF}_{\max } \sim 0.7$ ), we find that $\tau_{\text {cent }}=2$ days. This result suggests that there may be a small delay after all between the variations in the $B$ and $R$-band light curves. The positive centroid lag is due to the fact that at negative lags (down to $\sim-60$ days) the $F_{B}$ vs. $F_{R}$ DCF is smaller than the $F_{B}$ ACF while the opposite trend, i.e. $F_{B}$ vs. $F_{R}$ DCF being slightly larger than the $F_{B} \mathrm{ACF}$, is observed at the positive lags up to 60-70 days. Admittedly, this is a low amplitude effect, and longer light curves are necessary in order to test its validity.

\subsection{Correlation between flux and spectral variations}

We also investigated the cross-correlation between the fluxdensity light curves and the $F_{B} / F_{R}$ curve. In the bottom panel of Fig. 2 we plot the $\left(F_{B}+F_{R}\right) / 2$ vs. $F_{B} / F_{R}$ and the $F_{B}$ vs. $F_{B} / F_{R}$ DCFs (open circles and solid line, respectively; the $F_{R}$ vs. $F_{B} / F_{R}$ DCF is very similar to the ones plotted in this panel). Positive lags mean that variations in the flux-density light curve lead those in the flux ratio curve.

The two DCFs look very similar. They show a broad hump around zero time lag, with $\mathrm{DCF}_{\max } \sim 0.7$ in both cases. This result suggests that the flux and spectral variations are well correlated. This is not surprising, given the $F_{B} / F_{R}$ vs. $\left(F_{B}+F_{R}\right) / 2$ plot shown in the bottom panel of Fig. 1. The fact that the maximum DCF value is not larger than 0.7 can be explained by the significant, albeit low amplitude, scatter of the points around the best fitting PL model in this plot.

The most interesting aspect of both DCFs is the strong asymmetry towards negative lags. In order to highlight this effect, in the bottom panel of Fig. 2 we also plot the positive-lag $F_{B}$ vs. $F_{B} / F_{R}$ DCF curve reflected about the zero-lag axis (dashed line). It is now evident that the amplitude of the DCF at lags smaller than $\sim-10$ days is larger than that at the respective positive lags. 


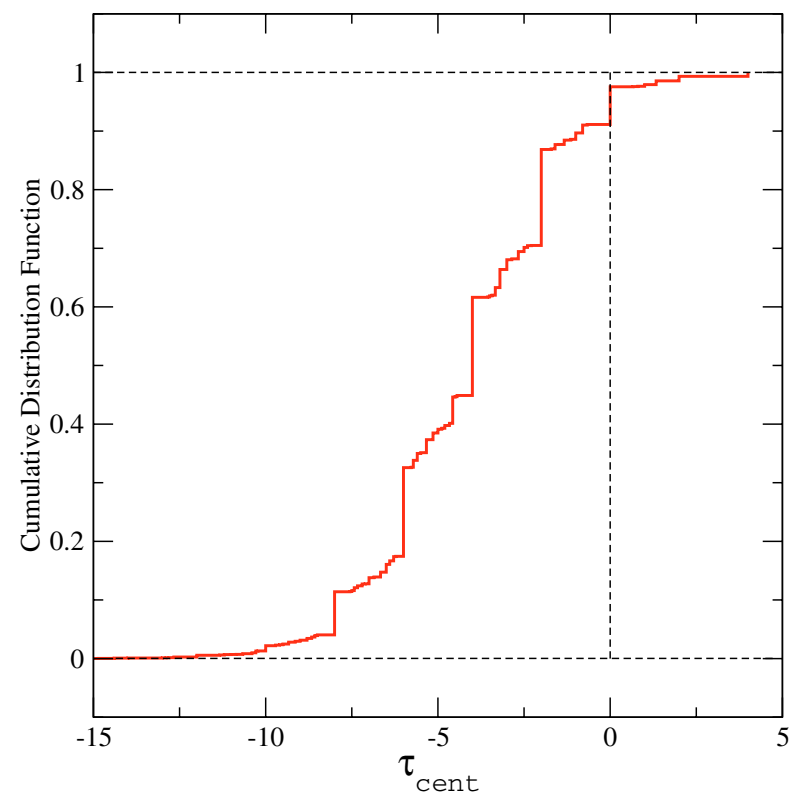

Fig. 3. The cumulative distribution function of $\tau_{\text {cent }}$ in the case of the $\left(F_{B}+F_{R}\right) / 2$ vs. $F_{B} / F_{R} \mathrm{DCF}$, as estimated using the results of the numerical experiment that we describe in Sect. 3.2.

This implies that there are delays between the flux and spectral variations, with the latter leading the former.

In order to quantify the average delay between the spectral and flux variations, we estimated the centroid of the $\left(F_{B}+\right.$ $\left.F_{R}\right) / 2$ vs. $F_{B} / F_{R}$ DCF using the same method as above. We found that $\tau_{\text {cent }}=-4$ days. In order to estimate the uncertainty in this measurement, we employed the bootstrap techniques of Peterson et al. (1998) and created 10000 pairs of simulated light curves. In Fig. 3 we plot the sample cumulative distribution of the centroid value as was estimated using the $\tau_{\text {cent }}$ values that we computed for the 10000 synthetic DCFs of our numerical experiment.

The average centroid value is equal to -4 days, and $68.3 \%$ of the synthetic DCFs yield centroid values between -6.4 and -2 days. Furthermore, only $2.4 \%$ of all DCFs resulted in $\tau_{\text {cent }}$ having a positive value. We conclude that the delay of $\sim 4$ days that we detect between the $F_{B} / F_{R}$ and the flux variations is significant.

The determination of the DCF centroid is based on a rather subjective method and depends on the number of DCF points involved in the calculation. Furthermore, the DCFs shown in the bottom panel of Fig. 2 show broad humps rather than well defined, narrow peaks. The broadness of the DCF maxima may imply the presence of more than just a single delay between the spectral slope and flux variations.

Having these remarks in mind, we conclude that, although the flux ratio $F_{B} / F_{R}$ follows rather well the increase/decrease of the source flux (see bottom panel in Fig. 1), the cross-correlation analysis reveals a subtle detail: $F_{B} / F_{R}$ decreases or increases before the respective flux changes. This result is significant at the $97.6 \%$ level. Our best estimate of the average delay between the flux and spectral slope variations is $-4_{-2.4}^{+2.0}$ days.

In order to further investigate this interesting issue, as well as the origin of the spectral variations, in the following section we focus our attention on two best sampled flares in the optical light curves shown in Fig. 1.
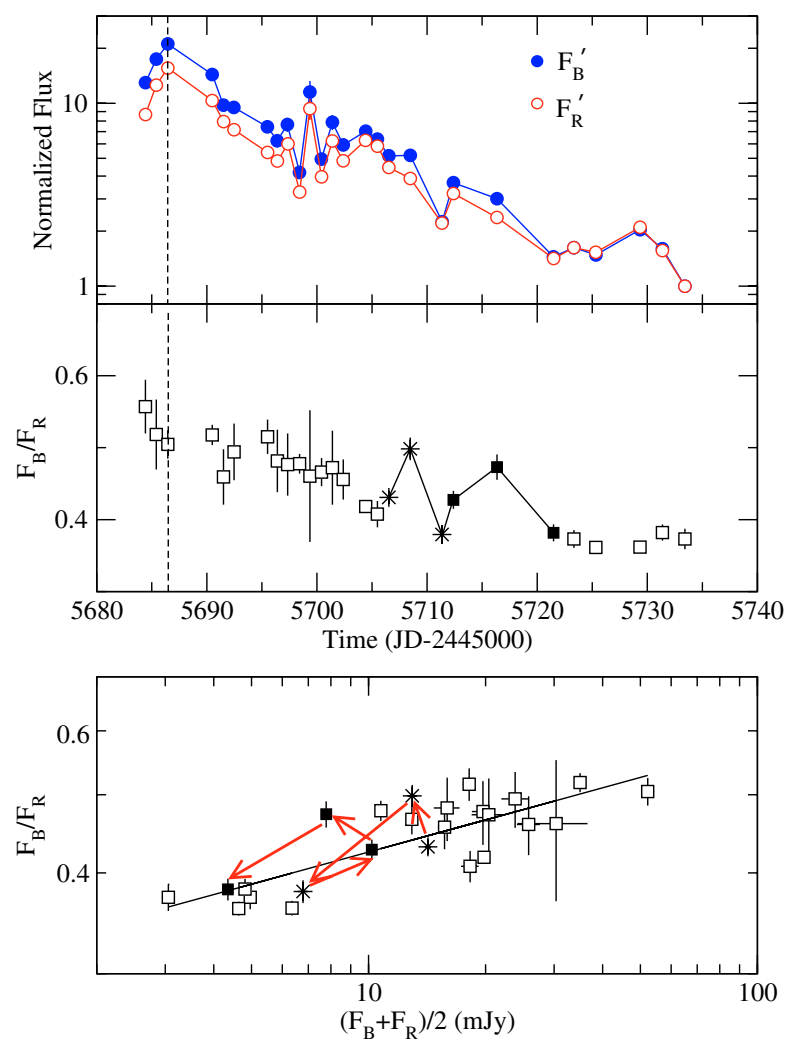

Fig. 4. Top panel: the last part of the $B$ and $R$-band light curves of the 1997 flare. The data are normalized to the flux density of the rightmost point. Middle panel: $F_{B} / F_{R}$ plotted as a function of time, using the data shown in the upper panel. The vertical line indicates the time of maximum flux. Some points are plotted with asterisks and filled squares for reasons explained in the text. Bottom panel: $F_{B} / F_{R}$ plotted as a function of $\left(F_{B}+F_{R}\right) / 2$. The solid line shows the best-fitting PL model to the data.

\section{Spectral analysis of individual flares}

\subsection{The 1997 flare}

The 1997 and 2001 large-amplitude optical flares of BL Lacertae are well sampled in the light curves that we use in this work (the respective data points in Fig. 1 are those in the time intervals 5650-5750 and 7050-7150).

In the top panel of Fig. 4 we plot the $B$ and $R$-band light curves corresponding to the last part of the 1997 flare (filled and open circles, respectively). The data sample the last few days of the high-brightness phase and the subsequent flux decline phase which lasted for $\sim 50$ days. We do not plot the data before JD = 2450680 because they are erratic and do not show any other well-defined flux rising or decaying parts. The light curves are normalized $\left(F_{B}^{\prime}, F_{R}^{\prime}\right)$ to their rightmost point (which, as a result, is set equal to one, for both of them), as this is their lowest flux point. In this way we can better compare variability amplitudes between the two light curves.

At the flare peak, the $B$ and $R$-band flux densities are $21.1 \pm$ 0.6 and $15.6 \pm 0.4$ times higher than the respective lowest levels. The difference between these two values (which represents the maximum variability amplitude of the light curves shown in Fig. 4) is $5.5 \pm 0.7$. However, as the flux decreases, this difference decreases as well. For example, $\sim 35$ days after the flare peak, the $B$ and $R$-band flux densities are only $1.45 \pm 0.04$ and $1.41 \pm 0.02$ times larger than the respective lowest points. From then on, the $B$ and $R$-band variability amplitudes are quite 
similar, indicating that the flux in the $B$ band decreased faster than the flux in the $R$ band.

In the middle panel of Fig. 4 we show the $F_{B} / F_{R}$ ratio, which is clearly variable. This result implies that the optical spectrum of the source changes, becoming "softer" ("redder") as the flux decreases. These spectral variations are due to the already noticed faster decrease of the $B$-band flux.

Interestingly, the data show that $F_{B} / F_{R}$ was decreasing even before the flux reached its maximum level. The vertical, dashed line in the top panels of Fig. 4 indicates the time of the flare peak. Although the errors of the two previous $F_{B} / F_{R}$ points are large, the data indicate that the flux ratio started decreasing in advance of the source flux. This behaviour is consistent with the " $\left(F_{B}+F_{R}\right) / 2$ vs. $F_{B} / F_{R}$ " DCF results that we reported in the previous section.

The bottom panel of Fig. 4 shows the " $F_{B} / F_{R}$ vs. $\left(F_{B}+\right.$ $\left.F_{R}\right) / 2$ " plot using the data that are plotted in the top panels of the same figure. The solid line shows the best-fitting PL model to the data. Its slope is $\sim 0.13$, almost identical to that of the best-fitting PL model to the data shown in the bottom panel of Fig. 1. We conclude that a constant difference between the variation rates in the two bands can produce spectral variations similar to the longterm, "mildly-chromatic" spectral variations that we observe in BL Lacertae between 1997 and 2002.

In the middle and bottom panels of Fig. 4 we have used different symbols for some points which appear to deviate significantly from the generally "smooth" trend of $F_{B} / F_{R}$ decreasing with time. Points plotted with asterisks and filled squares correspond to the flux "humps" that we observe in the time intervals 5707-5712 and 5712-5722, respectively. Although the flux variability amplitude of these "mini-events" is small, the associated spectral variations introduce significant scatter around the best-fitting PL model in the " $F_{B} / F_{R}$ vs. $\left(F_{B}+F_{R}\right) / 2$ " plot.

We may thus speculate that some of the scatter around the best-fitting PL model to the data shown in the bottom panel of Fig. 1 is introduced by short, low-amplitude events which are accompanied by spectral variations that do not follow the general trend of the larger-amplitude flares.

We note that there exists an interesting correlation between the spectral variations of these low-amplitude events and the respective flux changes. We have connected with arrows the points plotted with asterisks and filled squares in the " $F_{B} / F_{R}$ vs. $\left(F_{B}+F_{R}\right) / 2$ " plot of Fig. 4 to indicate the time evolution of these "mini-events" in this plane. We can see that these points define loop-like structures which evolve in the anti-clockwise direction.

\subsection{The 2001 flare}

During the BL Lac 2001 WEBT campaign, two large-amplitude flares, the first in mid 2001 and the second in early 2002, were detected (Villata et al. 2004b). The 2001 flare was doublepeaked. The second peak is well sampled in the WEBT light curves that we use in this work. The respective data are plotted in the top panel of Fig. 5. As in Fig. 4, we have normalized both light curves to the flux density of their rightmost point. In the middle panel we show $F_{B} / F_{R}$ as a function of time, and in the bottom panel the same quantity is plotted as a function of $\left(F_{B}+F_{R}\right) / 2$. The advantage of the 2001 light curves is that they sample well both the flux rising and flux decaying parts of the flare.

We fitted the rising and decaying parts of the light curves with an exponentially increasing, $F(t) \propto \exp \left[\left(t-t_{0}\right) / \tau_{\mathrm{r}}\right]$, and decreasing, $F(t) \propto \exp \left[-\left(t-t_{0}\right) / \tau_{\mathrm{d}}\right]$, function, respectively $\left(t_{0}\right.$ represents the time of the flare peak). The best-fitting models are
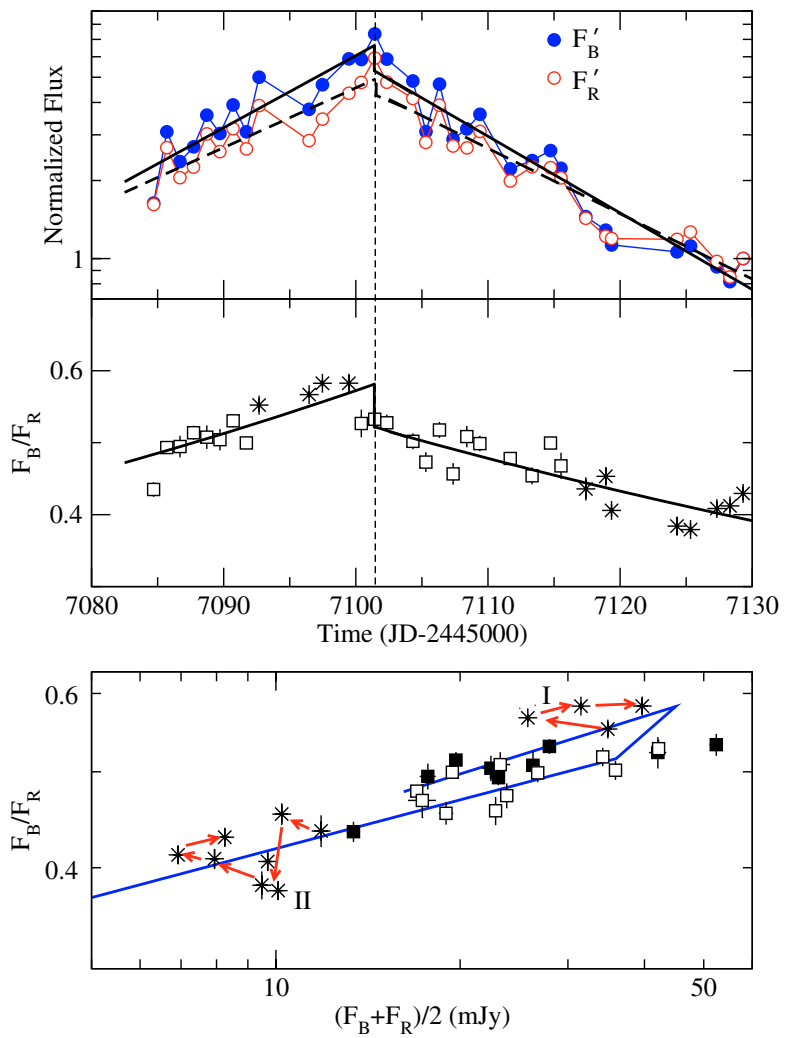

Fig. 5. Same as Fig. 4, but for the 2001 flare. The solid and dashed lines in the top panel show the best-fitting exponential functions to the $B$ and $R$-band data, respectively. The solid line in the middle panel shows the resulting $F_{B} / F_{R}$ ratio. Filled and open squares in the bottom panel show the data during the flux rising and decaying phases, respectively. Some points in the middle and bottom panels are plotted with asterisks for reasons explained in the text.

also plotted (normalized as the light curves) in the top panel of Fig. 5 (solid and dashed line for the $B$ and $R$-band light curves, respectively).

The model describes rather well the overall flux evolution, during both the rising and decreasing phases of the flare. Our best-fitting $\tau$ values are: $\tau_{\mathrm{r}, B}=16, \tau_{\mathrm{r}, R}=18, \tau_{\mathrm{d}, B}=13$, and $\tau_{\mathrm{d}, R}=15$ days (with an error of \pm 0.8 days in all cases). The differences between these best-fitting $\tau$ values suggest that: a) the flare is not symmetric, as the flux decrease is faster than the flux increase, i.e. $\tau_{\mathrm{d}}$ is smaller than $\tau_{\mathrm{r}}$ in both bands (by the same amount of $\sim 3$ days), and b) the flare evolves faster in the $B$ than in the $R$ band, as both the rise and decay $\tau_{B}$ values are smaller than the respective $\tau_{R}$ values by about 2 days.

The second result can explain the observed spectral variations. The solid line in the middle panel of Fig. 5 shows the $F_{B} / F_{R}$ curve derived from the best-fitting $B$ and $R$-band exponential functions. Obviously, the $F_{B} / F_{R}$ model curve fits the data well. At the beginning of the flare, the spectrum hardens as the flux increases, because the $B$-band flux increases faster than the flux in the $R$ band $\left(\tau_{\mathrm{r}, R}>\tau_{\mathrm{r}, B}\right)$. After the flare peak, the spectrum softens, because the $B$-band flux decreases faster than the $R$-band flux.

The vertical dashed line in the upper two panels of Fig. 5 indicates the time of the flare peak. One can see that the change of the spectral evolution from spectral hardening (i.e. $F_{B} / F_{R}$ increasing) to spectral softening $\left(F_{B} / F_{R}\right.$ decreasing $)$ happens a few days before the flux reaches its maximum. This is consistent with the DCF results that we report in Sect. 3.2. 
Furthermore, the data points in the "spectral slope vs. time" plot indicate that the change in the spectral evolution is rather abrupt. This is also clearly indicated by the significant "break" that appears in the $F_{B} / F_{R}$ model curve near the flux peak.

In the bottom panel of Fig. 5 we show the " $F_{B} / F_{R}$ vs. $\left(F_{B}+\right.$ $\left.F_{R}\right) / 2$ " plot. Filled and open squares correspond to the data during the rising and decaying phases, respectively. A PL model of slope $\sim 0.13$ (not plotted for clarity reasons), equal to that of the 1997 flare, describes well the " $F_{B} / F_{R}$ vs. $\left(F_{B}+F_{R}\right) / 2$ " relation.

The solid (blue) lines in the same panel show the "spectralslope vs. brightness" relation when we consider the best-fitting exponential rising and decaying functions, which are plotted in the upper panel of Fig. 5. In agreement with the data points (open squares lie systematically below the points plotted with filled squares), and because of the abrupt change of the spectral slope near the flare maximum, the "spectral-slope vs. brightness" model curve during the flux decay phase lies below the respective flux rise model curve.

Just like during the 1997 flare, low-amplitude flux variations introduce significant scatter in the " $F_{B} / F_{R}$ vs. $\left(F_{B}+F_{R}\right) / 2$ " plot. The points plotted with asterisks in the middle and bottom panels of Fig. 5 highlight the spectral variability behaviour of the source during such "events".

For example, during the time interval $7093-7102$ the flux decreased and then increased until the flare reached its peak. The respective points in the "spectral-slope vs. flux" plot follow a well defined loop, which evolves clockwise (loop "I"), as the arrows clearly show in the bottom panel of Fig. 5.

We observe loop-like patterns during the flux decaying part of the flare as well. The clearest example is the spectral evolution during the end of the flare, i.e. after $\sim 7117$. We can see that these points correspond to a flux decrease and a subsequent smooth flux increase. In the $F_{B} / F_{R}$ vs. $\left(F_{B}+F_{R}\right) / 2$ plot, the respective data, plotted with asterisks, follow a clockwise evolving loop-like pattern (loop "II"). Note that $F_{B} / F_{R}$ has already started increasing a few days before the flux increase.

\section{Discussion and conclusions}

BL Lacertae is one of the most frequently observed blazars, at all frequencies. It has been observed for more than a century in the optical band. The observations in the last years have been very intense, to a large extent due to the recent WEBT campaigns. The light curves that have resulted from these observations are densely sampled over a long time period ( $~ 8$ years). Although optical observations of the source will be carried out in the future as well, we believe that the current light curves are of sufficient quality (in terms of length and sampling) to allow the investigation of its "typical" variability characteristics.

Villata et al. (2004b) showed that the variations in the optical light curves of BL Lacertae can be interpreted in terms of a "strongly" and a "mildly-chromatic" component, which operate on short (i.e. $<1$ day) and longer time scales, respectively. They also showed that the short and longer time scale components determine a "bluer-when-brighter" slope of $\sim 0.4$ and $\sim 0.1$, respectively, in a $B-R$ vs. $R$ plot.

In this work, we continued the investigation of Villata et al. (2004b), and we studied in more detail the long-term, mildlychromatic spectral variations that these authors had already noticed. Our main results are as follows:

a) There is an indication that the $R$-band variations on time scales longer than a day are slightly delayed with respect to the variations in the $B$ band. However, this is a low amplitude effect and longer light curves are needed to confirm it. Even if real, this effect cannot explain the significant, long-term, optical spectral variations that we observe.

b) A constant difference between the rates which the flux rises/decays in the $B$ and $R$-band light curves can explain the spectral variations that we observe in BL Lacertae.

For example, we find that the 2001 flare data are consistent with exponentially rising/decaying functions. When the flux rises, $F_{B} / F_{R} \propto \exp \left[\left(t-t_{0}\right)\left(\tau_{\mathrm{r}, R}-\tau_{\mathrm{r}, B}\right) /\left(\tau_{\mathrm{r}, R} \tau_{\mathrm{r}, B}\right)\right]$. Using our bestfitting $\tau_{\mathrm{r}, R}$ and $\tau_{\mathrm{r}, B}$ values, we find that $F_{B} / F_{R} \propto F_{R}^{0.12}$. In the " $B-R$ vs. $R$ " plane this relation corresponds to a line of slope $\sim 0.12$, consistent with the findings of Villata et al. (2004b). We get a similar result when we consider the ratio $F_{B} / F_{R}$ during the flux decay phase. In this case, $F_{B} / F_{R} \propto F_{R}^{0.15}$.

Obviously, the rise and decay time scales, as well as the maximum variability amplitude, are not identical in all the "flarelike" events in BL Lacertae. In Fig. 1 one can detect many lowamplitude events, which last much less than the large-amplitude 2001 flare. What our results suggest is that the characteristic time scales of the flares cannot change arbitrarily from one to the other. As an example, if we assume exponentially rising/decaying flares, their time scales $\tau_{\mathrm{r} / \mathrm{d}, R}$ and $\tau_{\mathrm{r} / \mathrm{d}, B}$ should change in such a way that the ratio $\left(\tau_{\mathrm{r} / \mathrm{d}, R}-\tau_{\mathrm{r} / \mathrm{d}, B}\right) / \tau_{\mathrm{r} / \mathrm{d}, B}$ always remains roughly equal to $\sim 0.1$. In this way, the relation between the spectral slope and flux variations can be explained.

c) We find that the flux variations follow the spectral variations by an average delay of $\sim 4$ days. This is the first time that such an effect has been observed in BL Lacertae. A similar behaviour, in the optical band, has also been observed recently in 3C 66A (Böttcher et al. 2005). This effect can be clearly seen in the well-sampled 1997 and 2001 flares. The DCF results we report in Sect. 3.2 suggest that this delay operates most of the times in BL Lacertae.

If the "spectral-slope vs. brightness" plot can be explained by a constant difference between the variability time scales in the various bands (as we showed above), then the spectral hysteresis effect can be explained if one assumes that the variability time scale does not remain constant during the flare flux rise phase. The spectrum can start softening before the source reaches its maximum flux if the variability time scale, which determines the flux evolution, starts increasing before the peak at a rate that increases with frequency, or vice versa.

d) Although a power-law model describes well the "bluer when brighter" trend in the "spectral-slope vs. brightness" plot, it cannot provide a statistically acceptable fit to it. There exists significant scatter around the best-fitting model line.

To some extent, this scatter can be explained as the "remnant" of the fast, strongly-chromatic flares of Villata et al. (2004b) in the 1-day binned light curves we use. It is possible that in many cases the daily average points do not correspond to the "mean" of the fast flares but rather to a peak or a dip of the fast flares.

Our analysis in Sect. 4 shows, some of the scatter is also due to the fact that, occasionally, the spectral evolution of the source defines loop-like structures in the "spectral-slope vs. brightness" plot. In fact, if the ratio $F_{B} / F_{R}$ decreases/increases a few days before $F_{B}$ and $F_{R}$ (see result "c" above), then most flares that last for more than 5-10 days should show clockwise evolving loop structures in the $F_{B} / F_{R}$ vs. flux plot. This is what we observe during the 2001 flare.

Anti-clockwise evolving loops (like the ones observed in the flux decaying phase of the 1997 flare) imply that the spectral slope variations follow those in the flux light curves. The spectral-slope vs. flux DCF results suggest that these must be rare events. It will be interesting to investigate this issue in the 
future, using the even longer light curves that will be available then.

Thus, our two main findings are: i) the confirmation of the bluer-when-brighter mild chromatism of the BL Lac long-term optical variations, which is not caused by any delays between the $B$ and $R$-band light curves, but can be explained if the flux rise/decay rates in the two bands are different, by a fixed amount, and ii) the discovery that the spectral-slope variations tend to lead the flux variations, by a few days.

Villata et al. (2004b) explained the bluer-when-brighter behaviour in terms of a Doppler factor, $\delta$, variation on a convex spectrum. Indeed, an increase in $\delta$ implies both an increasing flux $\left(F_{v} \sim \delta^{3}\right.$; see e.g. Villata \& Raiteri 1999) and a change in the observed frequency $(v \sim \delta)$. The former effect causes the flux to increase at all frequencies, thus the appearance of a flare, while the intrinsic spectrum of the source is also "boosted" towards higher frequencies. If this spectrum is convex, we will observe a harder and harder part of it in our optical band, during the rising part of the flare, and vice versa during the dimming phase, i.e. the bluer-when-brighter trend. Thus, a change of $\delta$ in a convex spectrum makes flux variations in $B$ and $R$ bands happen simultaneously, but the $B$-band flux rises/decays faster than the $R$-band one.

Moreover, if the $\delta$ variation is due to a change of the jet viewing angle $\left(\delta=[\gamma(1-\beta \cos \theta)]^{-1}\right)$ and the jet is inhomogeneous and curved (see e.g. Villata et al. 2007), we will also have a tendency to a progressive reddening of the spectrum during the flare. Indeed, since the jet is inhomogeneous, it is composed of "slices" emitting different frequency ranges, which are redder going outwards along the jet curvature. Thus, as the jet approaches the line of sight, the $\delta$ variation will first affect the bluer and then the redder parts, implying a "reddening" of the spectrum. However, this reddening is opposed by the "bluer-when-brighter" trend during the flare rising phase. The combination of the two effects can result in a softening of the spectrum before the flare peak, as observed. In other words, the concomitance of the "bluer-when-brighter" trend with the progressive reddening, both of geometrical-kinematic origin, can (at least qualitatively) account for the changes in the spectral slope we analyzed in this paper.

A further observing as well as modeling effort is needed to clarify and quantify this interpretation. On the observational side, apart from BL Lacertae, the WEBT archive already stores long and well sampled light curves for three more sources, and more data will appear in the near future. The analayis of these light curves in a way similar to what we presented in this work, and the comparison of the results with those presented here, will hopefully help us understand better the variability mechanism in BL Lacs.

Acknowledgements. This work was partly supported by the European Community's Human Potential Programme under contract HPRN-CT-200200321 (ENIGMA).

\section{References}

Bevington, P. R. 1969, Data reduction and error analysis for the physical sciences (New York: McGraw-Hill)

Böttcher, M., Marscher, A. P., Ravasio, M., et al. 2003, ApJ, 596, 847

Böttcher, M., Harvey, J., Joshi, M., et al. 2005, ApJ, 631, 169

Edelson, R. A., \& Krolik, J. H. 1988, ApJ, 333, 646

Hu, S. M., Wu, J. H., Zhao, G., \& Zhou, X. 2006, MNRAS, 373, 209

Ostorero, L., Wagner, S. J., Gracia, J., et al. 2006, A\&A, 451, 797

Papadakis, I. E., Boumis, P., Samaritakis, V., \& Papamastorakis, J. 2003, A\&A, 397, 565

Peterson, B. M., Wanders, I., Horne, K., et al. 1998, PASP, 110, 660

Raiteri, C. M., Villata, M., Ibrahimov, M. A., et al. 2005, A\&A, 438, 39

Raiteri, C. M., Villata, M., Kadler, M., et al. 2006, A\&A, 459, 731

Ravasio, M., Tagliaferri, G., Ghisellini, G., et al. 2002, A\&A, 383, 763

Vaughan, S., Edelson, R., Warwick, R. S., \& Uttley, P. 2003, MNRAS, 345, 1271

Villata, M., \& Raiteri, C. M. 1999, A\&A, 347, 30

Villata, M., Raiteri, C. M., Kurtanidze, O. M., et al. 2002, A\&A, 390, 407

Villata, M., Raiteri, C. M., Aller, H. D., et al. 2004a, A\&A, 424, 497

Villata, M., Raiteri, C. M., Kurtanidze, O. M., et al. 2004b, A\&A, 421, 103

Villata, M., Raiteri, C. M., Balonek, T. J., et al. 2006, A\&A, 453, 817

Villata, M., Raiteri, C. M., \& Aller, M. F. 2007, ArXiv Astrophysics e-prints 